\title{
Side-effects of azathioprine treatment in rheumatoid arthritis: analysis of 10 years of experience
}

\author{
F SPEERSTRA, A M TH BOERBOOMS, L B A VAN DE PUTTE, H J VAN \\ BEUSEKOM,M W M KRUIJSEN AND J P VANDENBROUCKE
}

From the Department of Rheumatology, St Radboud Hospital, Nijmegen, and the Department of Epidemiology, Erasmus University, Rotterdam, The Netherlands

SUMMARY Our experience with azathioprine in the treatment of rheumatoid arthritis covers ten years, during which 91 rheumatoid patients ( 66 female and 25 male) received this drug, with a median treatment period of 36 months. Total follow-up experience, during and after treatment, was 399 person years. Twelve patients died. The principal causes of death were malignant neoplasm (six patients) and cardiovascular diseases (three patients). The mortality in our patients was compared to that of the general Dutch population by the Standardised Mortality Ratio (SMR). In the male patient group a significant excess of both total mortality and mortality from malignancy was observed. The female patients showed no differences from the general population. In this follow-up study, no lymphoreticular tumours occurred during or after azathioprine therapy.

Several clinical studies have reported improvement of articular symptoms in rheumatoid patients during azathioprine therapy. ${ }^{89} 95124143177224225$ After about 15 years of experience details of treatment and management during therapy are well defined. The question whether or not the risk of malignant growth in these patients is increased is still unanswered and remains of major concern in the prescription of this drug. The present follow-up study was undertaken because of the disquieting observation of a three hundred-fold increase in occurrence of lymphoproliferative tumours, with a particular cerebral localisation, in organ transplant patients who receive azathioprine among other immunosuppressive drugs. ${ }^{174}$

\section{Patients and methods}

Ninety-one patients (66 female and 25 male) with classical or definite rheumatoid arthritis, according to the American Rheumatism Association (ARA) criteria, ${ }^{186}$ were studied retrospectively. These patients were seen at the Department of Rheumatology of the St Radboud Hospital between 1 January
1970 and 31 December 1979. All suffered from active polyarthritis for which azathioprine therapy was instituted. From these patients data were collected retrospectively in 1980-1981 on age and sex, previous antirheumatic therapy, duration of arthritis, details of azathioprine treatment, survival and eventual cause of death. These data were available on all patients. The causes of death were determined either by direct communication with the general practitioner or from our medical records or from the death certificate. The mortality experience was compared with the mortality of the general Dutch population by means of the Standardised Mortality Ratio (SMR). ${ }^{9}$ Person years of follow-up were calculated from the start of azathioprine therapy up to the common closing date of the follow-up (31 December 1979), for males and females separately, and by five-year age categories. Death rates for the calculation of the expected mortality were obtained as an average of the general population death rates in the Netherlands during the follow-up period. For each of the sexes we took the average of the five-year age category specific death rates of three calendar years in the middle of the follow-up, that is, 1973, 1974 and $1975 .{ }^{165}$ The $95 \%$ confidence limits of the SMR were calculated under the assumption that the observed and the expected are realisations of a Poisson variable ${ }^{9}$ that can be transformed to approximate normality with constant variance by square root transformation. ${ }^{19}$

\section{Results}

Characteristics of this patient population are presented in table 1. Previous drug therapy to control the rheumatic disease had consisted of gold salts ( 83 patients), D-penicillamine (35 patients) or antimalarial agents (13 patients). Four patients had been treated with another cytostatic drug; cyclophosphamide (three patients) and chlorambucil (one patient). At the start of azathioprine therapy all patients were suffering from polyarthritis and 53 
TABLE 1 Characteristics of 91 rheumatoid patients $(25$ male and 66 female) treated with azathioprine. The data are expressed as median values

\begin{tabular}{lr}
\hline Duration of disease (yr) & 9 \\
Age at start of therapy (yr) & 54 \\
Duration of treatment (mth) & 36 \\
Total dose (g) & 120 \\
Follow-up (mth) & 60 \\
\hline
\end{tabular}

were receiving corticosteroids. The median duration of the disease was nine years (range one to 33 years). The daily dosage of azathioprine ranged from 1.5 to 2 $\mathrm{mg} / \mathrm{kg}$. In most cases the drug was used without interruption. With this regimen, some patients tolerated the drug for longer than ten years.

Side-effects consisted mainly of dose related reversible bone marrow depression, but were severe enough to necessitate discontinuation of therapy in nine patients. Intractable nausea occurred in four patients. Additional reasons for discontinuing therapy were severe bacterial infections (four patients) and herpes zoster (four patients). In five other patients, azathioprine was withdrawn because appropriate outpatient clinic supervision was not guaranteed.

The clinical response was judged ineffective in seven patients after at least three months of treatment. The majority experienced clinical improvement with long-lasting remission during continuous administration of azathioprine; half of the patients were on this regimen for more than three years.

Twelve patients died during the follow-up period (table 2). Necropsy was performed in five cases. Malignancies (six patients) and cardiovascular diseases (three patients) were the most common causes of death. The observed and the expected numbers were compared using the method already described. In the male group the difference in both 'total mortality' and 'all malignancies' was statistically significant at the $5 \%$ level (tables 3 and 4). No differences were

TABLE 2 Causes of death in 12 rheumatoid patients treated with azathioprine

\begin{tabular}{llll}
\hline & Sex & Age & Necropsy \\
\hline Malignant tumours & & & \\
Bronchial carcinoma & M & 64 & + \\
& M & 60 & + \\
Renal tumour & M & 69 & + \\
Breast carcinoma & F & 69 & - \\
Cardiovascular disease & F & 57 & - \\
$\quad$ Myocardial infarction & M & 67 & - \\
& M & 51 & - \\
Infectious diseases & M & 60 & + \\
Diverticulitis & F & 56 & + \\
Pneumonia & F & 81 & - \\
Other & & & \\
Uraemia/amyloidosis & F & 66 & - \\
\hline
\end{tabular}

TABLE 3 Comparison of mortality experience ('total mortality') (ICD 000-989) in male and female rheumatoid patients treated with azathioprine, with the values expected in the general Dutch population on the basis of the Standardised Mortality Ratio (SMR)*

\begin{tabular}{llclcl}
\hline & $\begin{array}{l}\text { Follow-up } \\
\text { person } \\
\text { years }\end{array}$ & $\begin{array}{l}\text { Observed } \\
\text { number } \\
\text { of deaths }\end{array}$ & $\begin{array}{l}\text { Expected } \\
\text { number } \\
\text { of deaths }\end{array}$ & SMR & $\begin{array}{l}95 \% \text { confidence } \\
\text { limits of SMR }\end{array}$ \\
\hline Males & 102 & 7 & $1 \cdot 9$ & $3 \cdot 7$ & $1 \cdot 4-7 \cdot 0^{\dagger}$ \\
Females & 297 & 5 & $3 \cdot 1$ & $1 \cdot 6$ & $0 \cdot 4-3 \cdot 3$ \\
Total & 399 & 12 & & & \\
\hline
\end{tabular}

*Death rates for the general Dutch population were obtained from published Dutch vital statistics for the calendar years $1973,1974,1975 .{ }^{165}$

tA significant increase at the $5 \%$ level in total mortality was observed in the group of male patients.

TABLE 4 Mortality in male and female rheumatoid patients treated with azathioprine. Comparison of the malignancies observed in the deceased with the values expected for 'All malignancies' (ICD 140-209) of the Dutch population on the basis of the Standardised Mortality Ratio $(S M R)^{*}$

\begin{tabular}{lllll}
\hline & $\begin{array}{l}\text { Observed deaths } \\
\text { from malignancies }\end{array}$ & $\begin{array}{l}\text { Expected } \\
\text { number } \\
\text { of deaths }\end{array}$ & SMR & $\begin{array}{l}95 \% \text { confidence } \\
\text { limits of SMR }\end{array}$ \\
\hline Males & 4 & 0.6 & $6 \cdot 7$ & $1 \cdot 7-15 \cdot 3+$ \\
Females & 2 & 0.9 & $2 \cdot 1$ & $0 \cdot 2-6 \cdot 2$ \\
Total & 6 & & & \\
\hline
\end{tabular}

*Death rates for the general Dutch population were obtained from published vital statistics for the calendar years $1973,1974,1975 .^{165}$

$\dagger$ The difference in the group of male patients is statistically significant at the $5 \%$ level.

found in the female group. In four male patients squamous cell bronchial carcinoma was diagnosed. Since malignant tumours of the respiratory tract, as well as cardiovascular disease, were the only causes of death in the male patients, we reviewed the patients' smoking habits from our medical records. An overwhelming majority (96\%) had a history of cigarette smoking, compared to $55 \%$ in the general male Dutch population. ${ }^{227}$

In the interpretation of our results it should be noted that one female patient had strong clinical evidence of malignant renal neoplasm (haematuria with massive tumour on intravenous pyelography), but necropsy was not performed. She was classified as a cancer death.

No differences with respect to total dose and duration of azathioprine therapy could be demonstrated between the patients who developed malignancies and those who did not. Seventy-nine patients are still alive and under medical outpatient care, which includes regular physical examination, blood counts, analysis of liver function and urine testing. $X$-rays of the chest and, if indicated, contrast radiological examinations have been performed. At the end of the follow-up period, no evidence of further malignancies had occurred in these patients, and no lymphoproliferative neoplasms have been diagnosed during or after azathioprine therapy. 


\section{Discussion}

For several years the clinical efficacy of azathioprine in the treatment of chronic inflammatory diseases, including rheumatoid arthritis, has been firmly established. Studies in rheumatoid patients, both in controlled $^{124143224225}$ and in open studies, ${ }^{89} 95177$ have revealed improvement of articular symptoms in the majority of patients. Moreover, reduction of corticosteroid dosage has been achieved in the first year of treatment. ${ }^{143}{ }^{177}$ Patients included in these studies were all suffering from long-standing active polyarthritis despite previous antirheumatic therapy.

In our study the majority of patients had previously been treated with either gold salts or D-penicillamine. Disease activity was reflected in the number of patients receiving corticosteroids $(58 \%)$. In accordance with earlier observations ${ }^{124} 143224$ improvement of articular symptoms was most pronounced in the first six months of treatment with azathioprine and could be maintained, to some degree, during long-term treatment with a dose of 1.5 to $2.0 \mathrm{mg} / \mathrm{kg}$ daily. Substantial reduction of corticosteroids (50\% or more) was effected in patients who tolerated the drug for at least six months.

Since long-term treatment is anticipated in rheumatoid patients, one might suspect that the cytotoxic and immunosuppressive properties of azathioprine may increase the risk of oncogenesis. Cytotoxic effects on bone marrow ${ }^{105}$ and the urogenital tract, ${ }^{1}$ as well as an increased number of chromosomal aberrations, have been reported ${ }^{95}$ but could not be confirmed by others. ${ }^{230}$ Whether a measurable degree of immunosuppression is achieved by low to moderate dosage regimens is uncertain. ${ }^{24} 208$ The high incidence of lymphomas, especially reticulum cell sarcoma, with intracerebral localisation, in patients with organ transplants is unprecedented. ${ }^{115}{ }^{174}$ Among other considerations, this fact focuses attention on the possible oncogenic effect of azathioprine in this category of patient. Nevertheless, the literature does not clearly show whether the occurrence of any particular malignancy in rheumatoid patients is increased in association with azathioprine therapy.

Two studies report a higher incidence of lymphomas, but both series included various chronic inflammatory conditions $\mathrm{s}^{115} 174$ and different cytostatic drugs were considered in one. ${ }^{174}$ Moreover, the risk of development of this type of tumour seems to be increased in rheumatoid patients ${ }^{101} 124$ and this fact should be taken into account.

Case reports dealing with the presumed oncogenic effect of azathioprine include leukaemia, ${ }^{32} 79$ adenocarcinoma of the lung ${ }^{177}$ and breast carcinoma ${ }^{177}$ Two cases are of special interest, because these patients developed malignant lymphoma with intracerebral localisation in both. ${ }^{127}{ }^{158}$ Our observations, covering 399 person years, revealed no lymphoreticular malignancies during or after azathioprine therapy. The excess mortality in the 25 male patients attracts attention, especially because four patients developed bronchial carcinoma.

In rheumatoid arthritis, death rates tend to be higher in the younger patients. ${ }^{3355125} 219$ Principal causes of death are cardiovascular disease, renal failure ${ }^{102} 156$ and, in earlier studies, infectious diseases. ${ }^{335}$ Two authors reported an increase of malignancies in their series, ${ }^{125}{ }^{151}$ but found no difference in the type of tumour between the cytostatic treated group and the control patients. ${ }^{64125}$ The increased number of squamous cell bronchial carcinomas found in our male patients may well be related to their smoking habits. Nevertheless, careful observation and follow-up of patients with rheumatoid arthritis receiving azathioprine should continue. Meanwhile this drug should only be given to those patients with severe rheumatoid arthritis who do not respond to, or cannot tolerate, other antirheumatic drugs. 